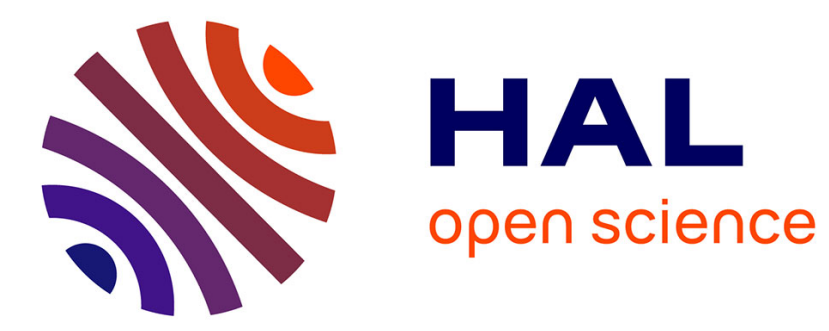

\title{
Positioning and Association Rules for Transparent Flying Relay Stations
}

Mehyar Najla, Zdenek Becvar, Pavel Mach, David Gesbert

\section{To cite this version:}

Mehyar Najla, Zdenek Becvar, Pavel Mach, David Gesbert. Positioning and Association Rules for Transparent Flying Relay Stations. IEEE Wireless Communications Letters, 2021, 10 (6), pp.12761280. 10.1109/LWC.2021.3063909 . hal-03220861

\section{HAL Id: hal-03220861 https://hal.science/hal-03220861}

Submitted on 9 Jun 2021

HAL is a multi-disciplinary open access archive for the deposit and dissemination of scientific research documents, whether they are published or not. The documents may come from teaching and research institutions in France or abroad, or from public or private research centers.
L'archive ouverte pluridisciplinaire HAL, est destinée au dépôt et à la diffusion de documents scientifiques de niveau recherche, publiés ou non, émanant des établissements d'enseignement et de recherche français ou étrangers, des laboratoires publics ou privés. 


\title{
Positioning and Association Rules for Transparent Flying Relay Stations
}

\author{
Mehyar Najla, Student Member, IEEE, Zdenek Becvar, Senior Member, IEEE, Pavel Mach, Member, IEEE, \\ and David Gesbert, Fellow, IEEE
}

\begin{abstract}
Transparent flying relay stations (FlyRSs), represented by transparent relays mounted on unmanned aerial vehicles (UAVs), have the potential to improve cellular networks capacity and coverage at little extra complexity and energy cost especially when compared with non-transparent relays. As the transparent relays do not transmit reference signals, they do not lend themselves easily to channel estimation. This makes solving the problems of user association and positioning of transparent FlyRSs much harder. We propose a solution enabling an efficient association of users to the FlyRSs and determining suitable positions of the FlyRSs. Surprisingly, this can be done knowing neither the qualities of the channels linking the FlyRSs and the users nor the users' location information. Our approach involves the users being grouped into clusters based on the channels to nearby static base stations via agglomerative hierarchical clustering. Then, 3D positions of one FlyRS per cluster are determined by deep neural networks. The proposal improves the users sum capacity with respect to existing solutions that rely on the knowledge of users positions.
\end{abstract}

Index Terms-Transparent relays, unmanned aerial vehicles, agglomerative hierarchical clustering, deep neural networks

\section{INTRODUCTION}

Relay stations (RSs) are deployed between a base station (BS) and a user equipment (UE) to improve the network's capacity and coverage [1]. Two main types of the RSs are distinguished: non-transparent (known also as Type I) and transparent (Type II) [1]. The non-transparent RSs create their own cell and perform typically all the functions that the BS does (e.g., radio resource management). In contrast, the transparent RSs are of limited functionalities and the BS still controls a majority of the communication management functions. Hence, the transparent RSs are less complex [2] and, consequently, lighter, cheaper, and less energy demanding comparing to the non-transparent RSs [3]. These features make transparent RSs convenient for flying relay stations (FlyRSs), i.e., the RSs mounted on Unmanned Aerial Vehicles (UAVs).

The FlyRSs can be deployed on-demand and can adjust their position to handle short-term traffic peaks [4]. However, the FlyRSs, in comparison to the fixed RSs, introduce new challenges related to the positioning of the FlyRSs [5] and the association of the UEs [6]. To solve these problems, the

This work has been supported by the grant no. P102-18-27023S funded by Czech Science Foundation. The work of David Gesbert was supported in part by the French government, through the 3IA Cte dAzur Project ANR-19P3IA-0002.

M. Najla, Z. Becvar, and P. Mach are with the Department of Telecommunication Engineering, Faculty of Electrical Engineering, Czech Technical University in Prague, Prague, 16627 Czech Republic (emails: najlameh@fel.cvut.cz; zdenek.becvar@fel.cvut.cz; machp2@fel.cvut.cz).D Gesbert is with Communication Systems Department, EURECOM, Sophia Antipolis, France (email: gesbert@eurecom.fr). locations of the UEs or at least the spatial distribution of the UEs are assumed to be known in [5], [6], and/or the quality of access channels between the FlyRSs and the UEs is obtained [7], [8], e.g., via an estimation from common reference signals. However, in practice, information on the UEs' locations is either inaccurate or not known at all due to the privacy preferences of the UEs. Moreover, the transparent FlyRSs do not transmit their own reference signals [9] and forward only data symbols transmitted by the BS [9] while the control and reference signals are sent to the UEs directly by the BS [9], [10]. Thus, the quality of the access channels between the FlyRS and the UEs is not known.

In the literature, two ways for the estimation of the access channel quality are proposed: i) the UEs feed their received downlink data signal back to the BS, which processes the fed-back signal [11], ii) the transparent RS listens to the uplink signal transmitted by the UEs and reports it to the BS [12]. However, such approaches imply a notable additional signaling and an undesired latency. The harm of this latency on the communication quality is emphasized if the FlyRSs are considered, as they can change positions dynamically and quickly over time. The positioning of the transparent FlyRSs and the association of the UEs to these transparent FlyRSs still remain unsolved for a practical case with unknown both locations of the UEs and the qualities of the access channels.

In this letter, we address the yet not solved problems of the transparent FlyRSs' positioning and the UEs' association in a practical urban scenario with unknown UEs' locations. We propose a solution that, first, clusters the UEs into disjoint groups so that the UEs with a high probability to be in LOS and in a proximity to each other are in the same cluster. As we target a realistic urban scenario with obstacles (e.g., buildings), the clustering is inspired by the Agglomerative hierarchical clustering algorithm [13]. Then, one FlyRS is deployed for each cluster and its 3D position maximizes the ratio of the access channels with LOS between the FlyRS and its associated UEs. The positions of the FlyRSs are predicted via offline trained deep neural networks (DNNs) exploiting the known qualities of the channels from the UEs to the BSs. This prediction benefits from the correlation between different types of channels and network's topology and environment. As this correlation is hidden and not explicitly available to be extracted analytically, we adopt DNNs, which are known to be efficient in extracting complex prediction models.

\section{System Model}

We assume an urban area with buildings and $M$ BSs deployed on the rooftops of some of the buildings. In addition, 


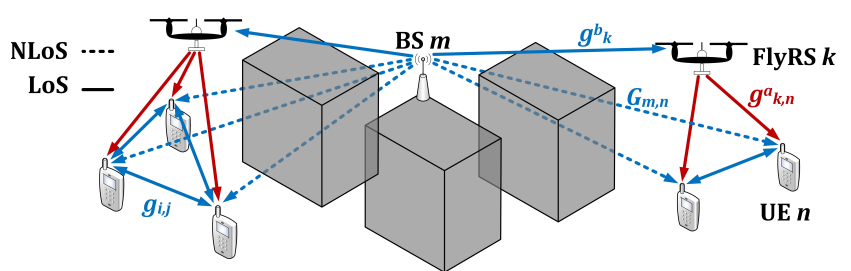

Fig. 1: System model. Red and blue lines denote unknown access and other known channels, respectively.

$K$ transparent FlyRSs relay data from the BSs to $N$ UEs in the downlink. The data is, first, sent via the backhaul channel from the BS to the FlyRS and, then, forwarded via the access channel from the FlyRS to the UE. Although every FlyRS is assumed to receive data from the BS providing the highest average signal to interference plus noise ratio, this assumption does not limit or restrict the proposed solution.

The FlyRSs operate in the half-duplex decode-and-forward mode. Thus, the FlyRSs transmit and receive data at the same frequency channel, but in a different time. Hence, the communication capacity of the $n$-th UE served by the $k$-th FlyRS relaying data from the $m$-th BS is determined as:

$$
C_{m, k, n}=\min \left(T^{b} C_{m, k}^{b},\left(1-T^{b}\right) C_{k, n}^{a}\right)
$$

where $C_{m, k}^{b}$ and $C_{k, n}^{a}$ are the capacities of the backhaul channel from the $m$-th BS to $k$-th FlyRS and the access channel from the $k$-th FlyRS to the $n$-th UE, respectively, and $T^{b} \in[0,1]$ is the relative duration of the transmission over the backhaul channel within one second. As shown in [14], the optimal $T^{b}$ is the one that equalizes $T^{b} C_{m, k}^{b}$ and $\left(1-T^{b}\right) C_{k, n}^{a}$ leading to $T^{b}=\left(C_{k, n}^{a}\right) /\left(C_{m, k}^{b}+C_{k, n}^{a}\right)$. Consequently, the FlyRS can forward only the data already received from the BS and such $T^{b}$ satisfies the information causality required for relaying [17]. Then, $C_{m, k, n}$ from (1) is defined as:

$$
C_{m, k, n}=T^{b} C_{m, k}^{b}=\left(1-T^{b}\right) C_{k, n}^{a}=\frac{C_{m, k}^{b} C_{k, n}^{a}}{C_{m, k}^{b}+C_{k, n}^{a}}
$$

The capacity of the backhaul channel between the $m$-th BS and the $k$-th FlyRS is derived as:

$$
C_{m, k}^{b}=B_{n} \log _{2}\left(1+\frac{P_{m} g_{m, k}^{b} \theta_{m, k}^{b} \phi_{m}}{B_{n} \sigma+I}\right)
$$

where $B_{n}$ is the bandwidth assigned to the $n$-th UE, $P_{m}$ is the transmission power of the $m$-th BS at the corresponding channel, $g_{m, k}^{b}$ is the backhaul channel gain between the $m$ th BS and the $k$-th FlyRS, $\theta_{m, k}^{b}$ is the fast fading power component, $\phi_{m}$ is the antenna gain of the $m$-th BS, $\sigma$ is the noise density, and $I$ is the interference from neighboring cells.

The capacity of the access channel between the $k$-th FlyRSs and the $n$-th UE is defined as:

$$
C_{k, n}^{a}=B_{n} \log _{2}\left(1+\frac{P_{k} g_{k, n}^{a} \theta_{k, n}^{a}}{B_{n} \sigma+I}\right)
$$

where $P_{k}$ is the transmission power of the FlyRS at the corresponding channel, $g_{k, n}^{a}$ is the access channel gain between the $k$-th FlyRS and the $n$-th UE. Note that $g_{k, n}^{a}$ is unknown due to the use of the transparent FlyRSs [3].

As in the conventional mobile networks, we consider that the network periodically estimates/measures the channel gains between any UE and the surrounding BSs [15], and the channel gain between the $m$-th BS and the $n$-th UE is denoted as $G_{m, n}$. Then, the channel gain between any two UEs is determined by the network exploiting the prediction presented in [16], where the authors prove that the channel gain between any two UEs can be predicted at a very low (or even no) additional signaling cost from the channel gains between those UEs and the surrounding BSs (i.e., from $G_{m, n}$ ) even in an urban area with buildings and dynamic obstacles (e.g., vehicles). The predicted channel gain between the $i$-th and $j$-th UEs is:

$$
g_{i, j}^{e}=g_{i, j}+E_{i, j}[d B]
$$

where $g_{i, j}$ is the true channel gain between the $i$-th and $j$ th UEs and $E_{i, j}$ is the statistical channel estimation error modeled as a zero-mean Gaussian error with a standard deviation of $e_{i, j}$, i.e., $E_{i, j}=\mathcal{N}\left(0, e_{i, j}\right)$ as in [16].

\section{PROBLEM FORMULATION}

The aim of this letter is to associate the UEs to the FlyRSs and to determine the positions of the FlyRSs in order to maximize the sum capacity of the UEs under the two following essential practical limitations: i) the qualities of the access channels are not known, as the FlyRSs are transparent, and ii) the locations of the UEs are not known. Then, the UEs sum capacity maximization problem is formulated as:

$$
\begin{array}{r}
\mathbf{X}_{\mathbf{K}}^{*}, \mathbf{Y}_{\mathbf{K}}^{*}, \mathbf{Z}_{\mathbf{K}}^{*}, \boldsymbol{\alpha}^{*}=\underset{\mathbf{X}_{\mathbf{K}}, \mathbf{Y}_{\mathbf{K}}, \mathbf{Z}_{\mathbf{K}}, \boldsymbol{\alpha}}{\operatorname{argmax}} \sum_{n=1}^{n=N} \sum_{k=1}^{K} \alpha_{k, n} C_{m, k, n} \\
\text { s.t. } \alpha_{k, n} \in\{0,1\} \forall k \in\{1,2, \ldots, K\}, \forall n \in\{1,2, \ldots, N\}(a) \\
\sum_{k=1}^{k=K} \alpha_{k, n}=1 \quad \forall n \in\{1,2, \ldots, N\}(b) \\
\left(x_{k}^{*}, y_{k}^{*}, z_{k}^{*}\right) \in \mathbf{A}^{\mathbf{o}} \quad \forall k \in\{1,2, \ldots, K\}(c) \\
z_{k}^{*} \in\left[z_{\text {min }}, z_{\text {max }}\right] \quad \forall k \in\{1,2, \ldots, K\}(d)
\end{array}
$$

where $\alpha_{k, n}=1$ if the $n$-th UE is associated to the $k$-th FlyRS and $\alpha_{k, n}=0$ otherwise, $\mathbf{X}_{\mathbf{K}}, \mathbf{Y}_{\mathbf{K}}$, and $\mathbf{Z}_{\mathbf{K}}$ are the $x, y$, and $z$ coordinates of all $K$ FlyRSs, respectively, $\alpha$ is the matrix of all binary association indicators $\alpha_{k, n}$ for all $N$ UEs and all $K$ FlyRSs, and $\mathbf{X}_{\mathbf{K}}^{*}, \mathbf{Y}_{\mathbf{K}}^{*}, \mathbf{Z}_{\mathbf{K}}^{*}$, and $\boldsymbol{\alpha}^{*}$ are the optimal $\mathbf{X}_{\mathbf{K}}, \mathbf{Y}_{\mathbf{K}}$, $\mathbf{Z}_{\mathbf{K}}$, and $\boldsymbol{\alpha}$, respectively. The constraints (a) and (b) guarantee that $\alpha_{k, n}$ is binary and each UE is associated to only one FlyRS, respectively. The constraint (c) guarantees that every FlyRS is positioned within the set $\mathbf{A}^{\mathbf{o}}$ of all possible locations in the area excluding buildings, obstacles, etc. The constraint (d) limits the altitude of the FlyRSs to a feasible/allowed range. Note that (6) targets to determine the FlyRSs' positions, but the trajectory optimization is out of the scope of this letter.

The problem (6) is an MINLP problem as $\mathbf{X}_{\mathbf{K}}, \mathbf{Y}_{\mathbf{K}}$, and $\mathbf{Z}_{\mathbf{K}}$ are continuous while $\boldsymbol{\alpha}$ is discrete. The MINLP problems are NP-hard and very difficult to solve analytically. Moreover, with unknown access channels and UEs' locations, the problem (6) cannot be solved analytically. Therefore, in the next section, we exploit clustering and DNNs to solve the association and positioning problems knowing neither the UEs' locations nor the qualities of the access channels. 


\section{PROPOSED POSITIONING AND ASSOCIATION RULES}

The proposed solution divides the UEs into disjoint clusters (subsection IV-A) and deploys one FlyRS per cluster (IV-B).

\section{A. Proposed UEs clustering}

The clustering of the UEs is inspired by the unsupervised Agglomerative Hierarchical Clustering, which groups the UEs that are similar to each other. In our case, two UEs are considered more similar to each other if the channel gain between these UEs is higher. This way, the UEs that are likely in LoS to each other are grouped into one cluster.

The proposed clustering starts with constructing a similarity matrix $\mathbf{S}$, which defines the similarity $S_{i, j}$ of every two UEs ( $i$-th and $j$-th $\mathbf{U E s}$ ) to each other. Hence, $\mathbf{S}$ is expressed as:

$$
\mathbf{S}=\left[S_{i, j}\right]_{N \times N} \text { where } S_{i, j}= \begin{cases}g_{i, j}^{e} & \text { if } i \neq j \\ 0 & \text { if } i=j\end{cases}
$$

where $\mathbf{S}$ is the zero-diagonal matrix, which includes the bilateral similarities (i.e., channel gains) among the $N$ UEs.

At the beginning of the proposed UEs' clustering, every UE represents a separated cluster. Then, in every step, the two clusters with the largest similarity are merged together. The similarity between two clusters is the average of all bilateral similarities between every pair of the UEs with each UE in the pair belonging to a different cluster. The algorithm stops when the largest similarity between two clusters becomes smaller than the threshold $g_{t h}^{e}$. We set the threshold as $g_{t h}^{e}=\mu \overline{\mathbf{S}}$, where $\overline{\mathbf{S}}$ is the average over all non-zero elements in $\mathbf{S}$, and $\mu$ is a constant. When the algorithm stops, the number of created clusters represents the number of FlyRSs to be deployed $(K)$. Hence, the lower $\mu$ is selected, the more FlyRSs are deployed and $\mu$ provides a trade-off between the number of deployed FlyRSs and the reachable sum capacity.

The result of the UEs' clustering is a set of clusters created so that the UEs in the same cluster are likely in LoS to each other. Hence, positioning one FlyRS to serve each cluster of UEs promises also LoS between the FlyRS and served UEs.

\section{B. Proposed 3D positioning of transparent FlyRSs}

In this section, we determine 3D positions of the FlyRSs. As stated in [17], the FlyRS's should be as close to the UEs as possible while avoiding the terrain and buildings. Taking this into consideration in addition to the fact that we have no information about the quality of the access channels or the UEs locations, determining $x, y$, and $z$ jointly is not possible. Hence, as in literature, see e.g., [18], we first determine $x$ and $y$ coordinates of the FlyRSs. The $x$ and $y$ coordinates are selected such that the ratio of the access channels with $\operatorname{LoS}$ is increased regardless of the FlyRS's altitude. Then, the altitude ( $z$ coordinate) of every FlyRS is optimized given the selected $x$ and $y$ coordinates, so that the FlyRS is as close as possible to the UEs while still keeping a sufficient backhaul capacity. This is done as follows.

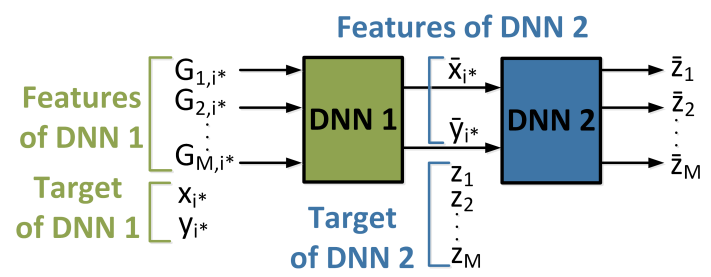

Fig. 2: Two DNNs for prediction of FlyRS's coordinates.

For every cluster $c$ containing $N^{c}$ UEs, the similarity submatrix $\mathbf{S}^{\mathbf{c}} \subset \mathbf{S}$ that includes the similarity $S_{i, j}^{c}$ between every two UEs from $c$ is created as:

$$
\mathbf{S}^{\mathbf{c}}=\left[S_{i, j}^{c}\right]_{N^{c} \times N^{c}} \text { where } S_{i, j}^{c}= \begin{cases}g_{i, j}^{e, c} & \text { if } i \neq j \\ 0 & \text { if } i=j\end{cases}
$$

where $g_{i, j}^{e, c}$ is the channel gain between the $i$-th and the $j$-th UEs from the cluster $c$.

Now, for every $i$-th UE from $c$, we derive the UE's average similarity (i.e., channel gain) to the other UEs in $c$ so that $\overline{\mathbf{S}_{\mathbf{i}}^{\mathbf{c}}}=\frac{1}{N^{c}} \sum_{j=1}^{N^{c}} S_{i, j}^{c}, \forall i \in\left\{1,2,, N^{c}\right\}$. This step corresponds to averaging the matrix $\mathbf{S}^{\mathbf{c}}$ over its columns, resulting in a row matrix $\mathbf{S}^{\mathbf{c}^{\prime}}=\left[\overline{\mathbf{S}_{1}^{\mathbf{c}}}, \overline{\mathbf{S}_{2}^{\mathbf{c}}}, \ldots, \overline{\mathbf{S}_{\mathbf{N c}}^{\mathbf{c}}}\right]$. Note that the probability that the $i$-th UE in $c$ has LoS channels with the other UEs in $c$ increases with rising $\overline{\mathbf{S}_{\mathfrak{i}}^{\mathbf{c}}}$. Therefore, in our case, $\mathbf{S}^{\mathbf{c}^{\prime}}$ expresses the probability distribution of the LoS at the channels from each UE in $c$ to the rest of the UEs in $c$. Hence, to increase the probability of LoS from the FlyRS to all served UEs, the FlyRS should be positioned over the UE with the highest $\overline{\mathbf{S}_{\mathfrak{i}}^{\mathbf{c}}}$, where $i \in\left\{1, \ldots, N^{c}\right\}$. The reason for such approach is that the UE with the highest $\overline{\mathbf{S}_{\mathbf{i}}^{\mathbf{c}}}$ has a high probability to be in LoS with other UEs in the same cluster.

We assume the realistic case with unknown locations of UEs. In such case, we can predict the location of the UE (denoted as $i^{*}$ ) above which the FlyRS should be positioned from the channel gains between this UE and the surrounding BSs, benefiting from the correlation between the location of the UE and the channels between this $i^{*} \mathrm{UE}$ and the surrounding BSs [16]. This task is hard to be solved analytically, especially with the presence of buildings randomizing the individual channel gains differently depending on the environment. Thus, we predict the coordinates of the FlyRSs via DNNs.

As explained above, we first determine $x$ and $y$ coordinates via DNN 1 and, then, these $x$ and $y$ coordinates are inserted to DNN 2 to determined $z$ coordinate as shown in Fig. 2 . Theoretically, if the FlyRS goes closer to the UEs and further from the static BS, the backhaul capacity decreases while the access channel capacity increases. At a certain height when the LoS between the FlyRS and the static BS changes to NLoS, the backhaul capacity decreases significantly. Thus, as we have no information about the access channels, DNN 2 is trained to select the lowest possible altitude while satisfying the constraints (c) and (d) in (6) and providing a sufficient backhaul capacity. For every FlyRS, we determine the altitudes $z=z_{1}, z_{2}, z_{M}$, where $z_{m}$ is the altitude of the FlyRS if this FlyRS is served by the $m$-th BS. Then, we select the $m^{*}$-th BS which provides the highest average SNR.

Both DNNs are trained offline (before the communication 


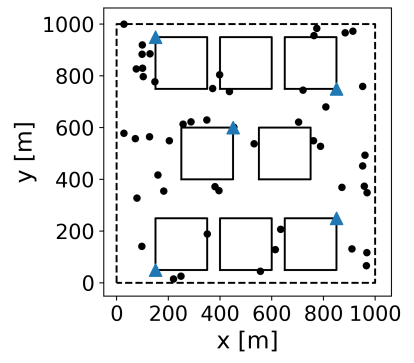

Fig. 3: Simulated scenario with building (black squares), BSs (blue triangles), and UEs (black dots).

sessions) in a supervised fashion. The training data includes features-target training samples. As the training is done offline, the samples can be both artificial (generated via simulations) or real data. For DNN 1, the features are represented by the channel gains between the UE and the surrounding BSs $\left(G_{1, i^{*}}, G_{2, i^{*}}, G_{M, i^{*}}\right.$ in Fig. 2) and the targets are the $x$ and $y$ coordinates of the FlyRS corresponding to the coordinates of the $i^{*}$-th UE ( $x_{i^{*}}$ and $y_{i^{*}}$ in Fig. 2) above which the FlyRS is positioned. As the $x$ and $y$ coordinates are continuous values, DNN 1 builds a regression model. Therefore, DNN 1 uses Mean Absolute Error loss function minimizing the absolute difference between the true and the predicted coordinates. The predicted $x$ and $y$ coordinates represent features of DNN 2 that predicts a set of the FlyRS's $z$ coordinates. The DNN 2 is trained with the targets obtained via an exhaustive search over a suitable range of a finite set of the altitudes to build a classification model. Hence, DNN 2 uses the cross entropy loss function minimizing the number of miss-classifications.

For both DNNs, every batch of training samples is inserted to the DNN with the initialized weights and biases flowing from the first layer to the last layer (i.e., the output layer). Then, the weights and the biases of every layer are updated sequentially and backwards (i.e., using backpropagation) repeatedly until the number of training iterations is reached. After the training, the DNNs predict the 3D position of the FlyRS online (i.e., during the communication session).

\section{Performance Analysis}

We consider a square urban area with buildings and up to 300 UEs (see Fig. 3). The height of each building is set uniformly from 20 to $30 \mathrm{~m}$. Furthermore, five BSs are deployed on the rooftops of the buildings. The path loss models of the LoS communication channels are in line with [19]. For the NLoS channel, an attenuation of $20 \mathrm{~dB}$ per every wall is added to the LoS path loss. The LoS and NLoS status of the channel is determined directly from the deployment of buildings and their mutual positions with respect to the positions of UEs and FlyRSs. Both LoS as well as NLoS channels include fast fading power components generated as exponentially distributed random variables with unit mean. We consider also interference from neighboring cells modeled randomly via Gaussian distribution $\mathcal{N}(-90,10)$. As the bandwidth allocation does not influence the proposed solution itself, we split the band equally among the UEs.

For the proposed UEs' clustering algorithm, the constant $\mu$ defining the stopping threshold (see Section IV-A), is set experimentally to $0.9,0.88,0.86,0.84,0.82$, and 0.8 for 50 , $100,150,200,250$, and 300 UEs, respectively. These values of $\mu$ are chosen to keep a relatively low and acceptable number of deployed FlyRSs, i.e., 5.3, 6.2, 6.9, 7.6, 8.3, 9.1 FlyRSs are in average deployed for the respective numbers of the UEs. The decreasing $\mu$ with the increasing number of UEs allows to deploy more FlyRSs when more UEs are present (as explained in Section II). Note that more analysis related to the detailed setting of $\mu$ and its effect on the number of deployed FlyRSs is left for future work due to the space limit.

Both DNNs (DNN 1 and DNN 2) are fully-connected and composed of three hidden layers with 30,40 , and 40 neurons, respectively. For all three hidden layers, we use the common rectified linear unit (ReLU) activation function. The number of learning samples is $10^{6}$ and $80 \%$ of the samples are used for the training and $20 \%$ for testing. The weights and the biases of DNNs with a batch size of 1000 samples are updated by stochastic gradient descent. The Xavier initialization is adopted to initiate the weight of DNNs. The number of training epochs is set to 5000 and the learning rate is set to 0.001 with a customized decay to 0.0001 after the 2000-th epoch.

We compare our proposed solution to the well-known Kmeans algorithm exploited, e.g., in [20], for the joint positioning of the FlyRSs and the association of the UEs. Note that K-means requires the knowledge of the UEs' locations and also the number of FlyRSs should be known in advance. Such knowledge required by K-means is usually not available in the real networks as the locations of at least some of the UEs may be unknown and the number of required FlyRSs varies with the number of present UEs. Thus, K-means implies requirements that cannot be fulfilled, and we include K-means in the simulations only to benchmark the performance of our proposal that eliminates these unrealistic requirements. Note that we set the number of FlyRSs for K-means to the number required by our proposal (i.e., $K$ ) for a fair comparison.

To show the effect of the possible error in the estimation of the channels between the UEs (see (5)), we present the results of our proposal when the channel gains among the UEs: i) are perfectly known, i.e., $E_{i, j}=0$ in (5) (denoted as Proposal without channel estimation error) and ii) are estimated with the zero-mean Gaussian error with the standard deviation set such that $\left(g_{i, j} / e_{i, j}\right)_{d B}=10 \log _{10}\left(\frac{g_{i, j}}{e_{i, j}}\right)=10 \mathrm{~dB}$, see (5), labeled as Proposal with channel estimation error.

In addition to K-means and two variants of the proposal, we also show an additional scheme, which divides the UEs into disjoint groups using the proposed UEs' clustering and, then, a single FlyRS is deployed in the center of gravity (COG) of every cluster (this scheme is denoted as Proposed clustering + COG positioning). Note that, the locations of the UEs should be known to determine the COG. This scheme is presented to show gains achieved by the proposed clustering with a common existing positioning, which however assumes unrealistic knowledge of accurate UEs' positions.

Last, we also show the performance of the proposed scheme when channel gains among the UEs are known perfectly and the FlyRSs are positioned exactly above the chosen UE in every cluster with optimal altitude derived via exhaustive 


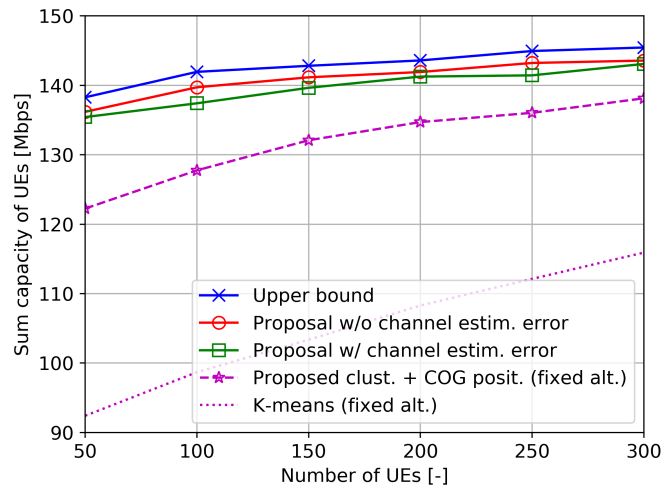

Fig. 4: Sum capacity of the UEs served by the FlyRSs

search (without the DNN-based prediction). This case, denoted as Upper bound, indicates a theoretical maximal performance.

Fig. 4 shows the sum capacity of the UEs versus different numbers of UEs $(N)$ in the area. The sum capacity of all simulated algorithms increases with the number of UEs. This behavior is justified by the increase in the number of deployed FlyRSs (as indicated above in this section) if more UEs are present in the area. Fig. 4 also shows that the Proposal without channel estimation error is only up to $3 \%$ below the Upper bound. This confirms the accuracy of the regression model built by the DNNs. Moreover, in Fig. 4, we see that the Proposal with channel estimation error loses less than $2 \%$ comparing to the Proposal without channel estimation error. This demonstrates the high robustness of the proposal against possible errors in the estimation of the channels among the UEs. This robustness results from the fact that the steps and calculations related to the proposed approach $\left(g_{t h}^{e}\right.$ and $\mathbf{S}^{\mathbf{c}^{\prime}}$ in Sections IV-A and IV-B, respectively) are designed to average out the errors in the estimation of the channels among UEs.

In addition to the robustness, Fig. 4 illustrates the superiority of the proposal compared to K-means in terms of the UEs sum capacity. The proposed clustering algorithm itself (Proposed clustering + COG positioning) introduces a gain in the sum capacity between $19 \%$ and $32 \%$ compared to K-means. Moreover, the Proposal with channel estimation error including the proposed clustering and positioning increases the sum capacity gain to the range from $23 \%$ to $46 \%$ comparing to K-means. This gain results from the fact that the proposal increases the LoS ratio at the access channels between the FlyRSs and the UEs comparing to K-means. This is confirmed in Fig. 5 showing the LoS ratio at the access channels (i.e., the number of UEs with LoS channels to the serving FlyRS divided by the total number of UEs). Fig. 5 confirms that the general pattern of the LoS ratio is analogical to the sum capacity pattern shown in Fig. 4 for all algorithms. Fig. 5 affirms that the full proposal including both clustering and positioning increases the LoS ratio at the access channels comparing to K-means by $27 \%$ to $46 \%$.

The time complexity of our proposal is $\mathcal{O}\left(N^{2} \log N\right)$ and it is given mainly by the agglomerative hierarchical clustering with average linkage. Such complexity is still low in terms of practical implementation of our proposal even for a high density of UEs.

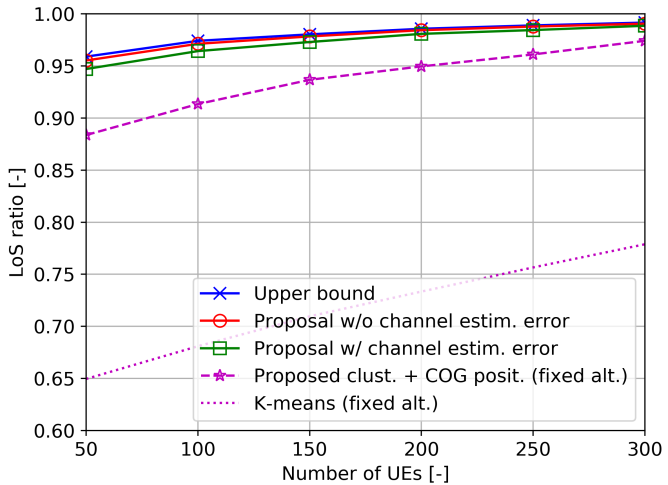

Fig. 5: LoS ratio vs number of UEs.

\section{CONCLUSION}

We have proposed a new scheme for 3D positioning of transparent FlyRSs and UEs' association. The proposed solution enables to perform these tasks with the knowledge of neither the qualities of the channels between the FlyRSs and the UEs nor the UEs' locations. Besides enabling the practical use of the transparent FlyRSs in the realistic scenarios, the proposal increases the UEs' sum capacity even with respect to the solution based on the known locations of the UEs.

\section{REFERENCES}

[1] C. Hoymann, et al., "Relaying operation in 3GPP LTE: challenges and solutions," IEEE Commun. Mag., vol. 50, no. 2, pp. 156-162, Feb. 2012.

[2] Huawei, "Understanding on Type 1 and Type 2 Relay," 3GPP TSG RAN WG1 Meeting \#57bis, Los Angeles, USA, June 29 - July 3, 2009 (R1 092370).

[3] M. Najla, et al., "Integrating UAVs as Transparent Relays into Mobile Networks: A Deep Learning Approach," in IEEE PIMRC, 2020, pp. 1-6.

[4] B. Galkin, et al., "UAVs as mobile infrastructure: Addressing battery lifetime," IEEE Commun. Mag., vol. 57, no. 6, pp. 132-137, Feb. 2019

[5] J. Chen, et al., "Optimal positioning of flying relays for wireless networks: A LoS map approach," in IEEE ICC, May 2017, pp. 1-6.

[6] M. Mozaffari, et al., "Optimal transport theory for cell association in UAV-enabled cellular networks," IEEE Communications Letters, vol. 21, no. 9, pp. 2053-2056, May 2017.

[7] Y. Zeng and R. Zhang, Energy-Efficient UAV Communication with Trajectory Optimization, IEEE Transactions on Wireless Communications, vol. 16, no. 6, pp. 3747-3760, Mar. 2017.

[8] M. D. Nguyen, et al., "UAV Trajectory and Sub-channel Assignment for UAV-based Wireless Networks," in IEEE WCNC, May 2020, pp. 1-6.

[9] R. N. Braithwaite, et al., "Improving data throughput for cell-edge users in a lte network using up-link harq relays," in IEEE VTC Fall, Sep. 2011, pp. $1-5$.

[10] R1-100951, ALU, ALU Shanghai Bell, CHTTL, Type 2 relay summary, RAN1 \#60, San Francisco, CA, Feb. 2010.

[11] D. Kim, et al., Filter-and-forward transparent relay design for OFDM systems, IEEE Transactions on Vehicular Technologies, vol. 62, no. 9, pp. 4392-4407, May 2013.

[12] J. Sydir and R. Taori, An evolved cellular system architecture incorporating relay stations, IEEE Commun. Mag., vol. 47, no. 6, pp. 115-121, Jun. 2009.

[13] S. C. Johnson, Hierarchical clustering schemes, Psychometrika, vol. 32, no. 3, pp. 241-254, 1967

[14] P. Mach, et al., "Joint Association, Transmission Power Allocation and Positioning of Flying Base Stations Considering Limited Backhaul," in IEEE VTC FalL, 2020, pp. 1-7.

[15] D. Astely, et al., "LTE: the evolution of mobile broadband" IEEE Communications Magazine, vol. 47, no. 4, pp. 44-51, May 2009.

[16] M. Najla, et al.,"Predicting Device-to-Device Channels from Cellular Channel Measurements: A Learning Approach ," IEEE Transactions on Wireless Communications, vol. 19, no. 11, Jul. 2020. 
[17] Y. Zeng, et al., "Throughput maximization for UAV-enabled mobile relaying systems," IEEE Transactions on Communications, vol. 64, no. 12, pp. 4983-4996, Sep. 2016.

[18] Y. Sun, et al., "Location optimization and user association for unmanned aerial vehicles assisted mobile networks," IEEE Transactions on Vehicular Technology, vol. 68, no. 10, pp. 10056-10065, Aug. 2019.

[19] 3GPP: 36.814 - Evolved Universal Terrestrial Radio Access (E-UTRA) Further advancements for E-UTRA physical layer aspects, v 9.2.0, 2017.

[20] B. Galkin, et al., "Deployment of UAV-mounted access points according to spatial user locations in two-tier cellular networks," Wireless Days, Mar. 2016, pp. 1-6. 